\title{
THE PHYSIOTHERAPY OF FLEXOR TENDON INJURIES TO THE HAND
}

\section{J. DU PLESSIS, B.Sc. in Physiotherapy (Stell.)*}

Of the 92 non-European and 32 European hand patients referred to our Department for Physiotherapy during the six month period January to June 1978, $31 \%$ and $34 \%$ respectively had suffered flexor tendon injuries, with or without nerve and blood vessel involvement. These formed the majority of our,patients, since any other single type of hand injury amounted to under $10 \%$ of the total number of patients treated.

The causative factors will not be discussed, but this article will be confined to the description of physiotherapy treatment of flexor tendon injuries following the types of surgical repair described in the preceding article by $\mathrm{L}$. K. Pretorius.

General considerations to be taken into account when treating tendon injuries are: (1) age; (2) infection; (3) associated injuries e.g. nerves, fractures; (4) mentality of patients; (5) occupation.

Young patients normally do very well, but with elderly patients or patients whose injury has been complicated by sepsis, treatment must be carried out very carefully and may have to be modified to suit the needs of a particular patient.

\section{PHYSIOTHERAPY FOLLOWING THE BUNNELC- TYPE REPAIR WITH BUTTON \\ (Zone I - II)}

The hand and forearm are placed in a plaster back-

\footnotetext{
* Physiotherapist, Tygerberg Hospital, Parowvallei.
}

slab with the wrist in slight flexion and the metacarpophalangeal (MP) joints in at least $90^{\circ}$ flexion. A cling bandage fits into the palm, keeping the fingers in a position of semi-flexion.

\section{2 weeks:}

No mobilization is carried out save for the sligy active extension and flexion of the fingers allowed by the plaster of Paris (POP) and cling bandages respectively.

\section{2 - 4 weeks:}

At this stage, the cling bandage is removed and active extension of the fingers to the limit of the plaster slab is performed. More flexion is now possible but no resistance is allowed. Specific passive physiological flexion and extension are given to the individual interphalangeal (IP) joints, whilst keeping the relevant MP and other IP joints in as much flexion as possible when doing the extension. At no time must any stretch be afforded the tendon and each passive movement must be localized to that particular joint. It is advisable to be conservative with passive movement at this stage. (See Fig 1.1 and 1.2.)

\section{4 - 6 weeks:}

The plaster slab is removed whilst the suture and button are retained. Gentle mobilizing oil massage is applied to the scar on the finger. No passive stretch is as yet applied to the limited joints. One now aims for 


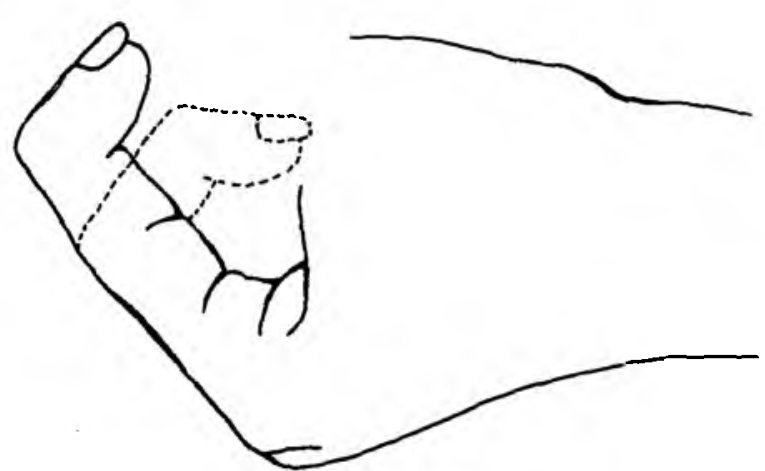

Fig. 1.1. Passive flexion and extension of P.I.P. joint maintaining M.P. and D.I.P. joint flexion.

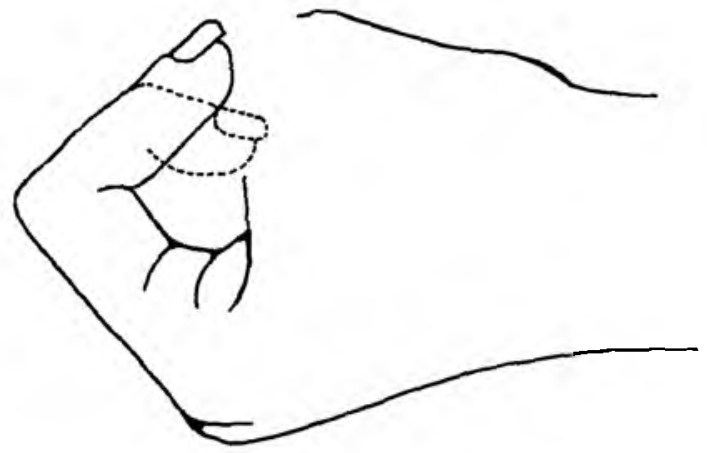

Fig. 1.2. Passive fiexion and extension of D.I.P. joint maintaining M.P. and P.I.P. joint flexion.

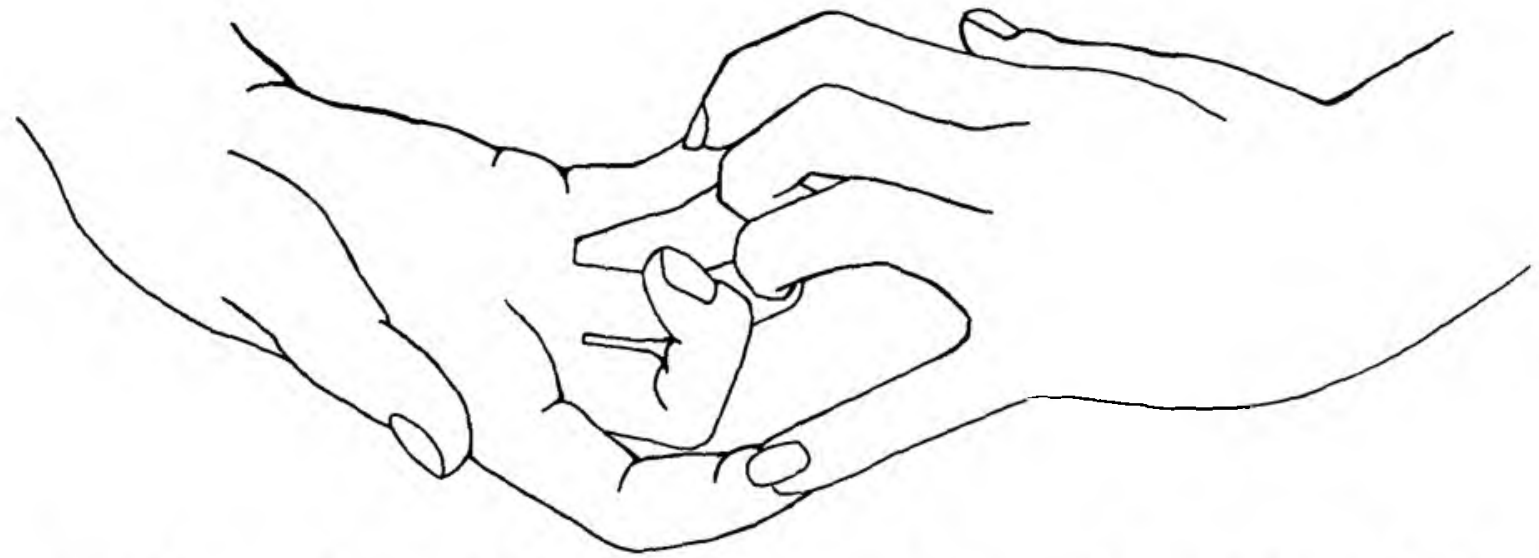

Fig. 2. Associated movement of Flexor Digitorum Profundus by resisting movement of normal fingers.

further active flexion through the inner range without giving resistance, although minimal resistance may be given to the distal phalanges. This is purely to act as a guide for the movement required. If patients have difficulty in flexing the distal interphalangeal (DIP) inints, strong resistance may be given to flexion of le unaffected fingers. Since flexor digitorum profundus (FDP) has a mass action, this will also encourage the injured finger to move (See Fig. 2).

Frequency of treatment: Daily treatment is impractical since the patients are all out-patients. Provided the patient is motivated and understands the need for exercising at home between sessions and the physiotherapist is satisfied that he will not overexercise, treatment is given three times per week.

\section{6 weeks onwards:}

This will be discussed under the corresponding period after the Kleinert-type of postoperative treatment, since treatment principles are similar.

\section{THE KLEINERT-TYPE OF POSTOPERATIVE} REGIME

\section{0 - 2 weeks:}

Immediately postoperatively, or a few days after suturing, elastics are inserted through the nails at the finger tips and attached by means of a plaster to the forearm, keeping the fingers in semi-flexion. Exercise is commenced immediately the elastics are applied. Patients are encouraged to do active finger extension against the elastics up to the limit of the POP. They then relax the extensors and let the tension on the elastics pull the fingers back into flexion. Since no active flexion is allowed as yet, careful patient instruction is essential.

The MP and IP joints must, however, be kept mobile. This is achieved by putting the MP joints in as much flexion as possible for extension of the IP joints and then doing full range passive physiological movements of the IP joints individually (Fig. 3.1 and 3.2).

With injuries in Zone III and IV, there is a strong likelihood of adhesions forming between the tendons and scar tissue. It therefore helps to extend and relax fingers individually, thus allowing the tendons to glide past each other.

Frequency of treatment:

Treatment is given three times per week depending on the co-operation of the patient and the mobility of the fingers.

\section{2-4 weeks:}

Treatment is continued as above, but the patient may now be asked to flex his fingers from the relaxed semi-flexed position. This is merely to test the tendons for functional ability.

\section{4-6 weeks:}

The surgeon usually decides to remove the plaster slab, but retains the elastics for a further two weeks. 1. For treatment purposes only, the elastics may now 


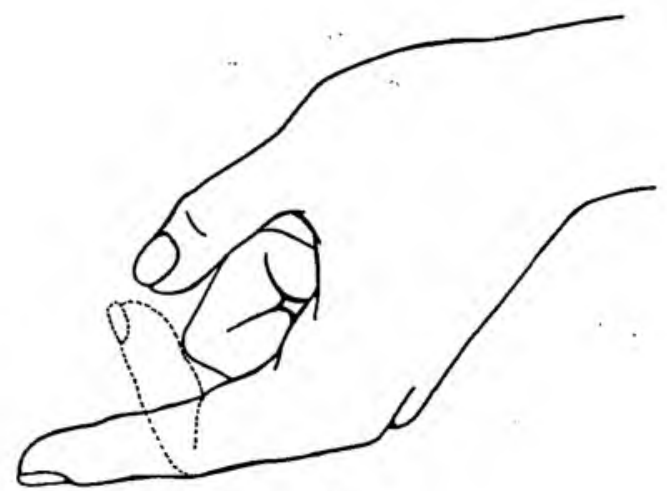

Fig. 3.1. Passive P.I.P. joint flexion maining M.P. joint fexion.

be removed. A soap and oil massage with the arm in elevation is given to remove the dead skin and increase the blood flow, keeping the wrist and fingers flexed.

2. Gentle massage, using olive oil, is given over the scar tissue to increase tissue mobility.

3. A low dose of ultrasound $\left(1 \mathrm{~W} / \mathrm{cm}^{2}\right.$ pulsed for 5 minutes) is given over the scar, again to improve tissue mobility.

4. Passive and active movements now include simultaneous extension of PIP and DIP joints, whilst retaining MP flexion.

5. Active finger flexion is permitted. We find that a softly inflated plastic ball/tube is useful, for it provides the required amount of resistance needed to guide the movement. Patients may experience difficulty in performing active flexion which may be overcome by:

5.1. Demonstration and explanation of function on the unaffected hand,

5.2 Application of strong resistance to uninvolved fingers of the injured hand whilst encouraging contraction of sutured tendons by giving minimal resistance to flexion of the injured finger purely as a guide for movement.

6. If both FDP and FDS tendons to the same finger have been sutured, then it is important to elicit the specific functions of these tendons i.e. flexion of DIP joint and then flexion of PIP joint whilst DIP joint remains extended. Surgically, if both tendons to a finger are severed, one is often sacrificed.

7. Wrist mobilization is started, using passive joint mobilization techniques according to Maitland and Kaltenborn. These techniques are modified for each particular patient's need; all the fingers are always maintained in flexion during mobilization.

6 weeks onwards (for both types of suture):

The tendons should now be strong enough for the physiotherapist to go ahead and achieve full joint mobilization of both the wrist and fingers. The patient is also referred for occupational therapy at this stage for functional re-education in the finer hand skills.

Aims of physiotherapy are to:

1. Gain full active use of the individual fingers.

2. Gain full functional simultaneous use of all the fingers i.e. the hand as a whole.

3. Loosen adhesions.

4. Mobilize scar tissue.

5. Mobilize joints.

6. Ensure the fullest possible functional use of the hand. If there is no likelihood of further improve-

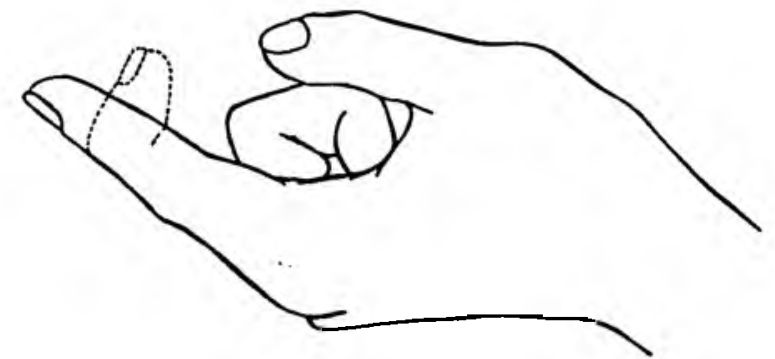

Fig. 3.2. Passive D.I.P. joint flexion and extension maintaining M.P. joint flexion.

ment, the necessary referrals to the occupation therapist and/or work placement officer should $\mathrm{k}$ ? made.

\section{TECHNIQUES}

1. Wax baths.

2. Scar tissue massage.

3. Proprioceptive neuromuscular facilitation (PNF) 3.1 Hold-relax techniques

3.2 Repeated contractions

3.3 Rhythmic stabilizations.

4. Ultrasound

5. Passive manual stretching of joint structures and two-joint muscles.

6. Serial plasters.

7. Faradism under tension (occasionally necessary where wrist adhesions persist)

8. Passive joint mobilizing techniques according to Maitland and Kaltenborn for peripheral joints.

9. Home exercise programme.

Specific points with regard to techniques $1-8$ :

1. Wax baths are useful before treatment as a means of softening scar tissue and relaxing the hand. Patients are instructed how to use the wax bath and do this on their own whilst the physiotherapist treats other patients.

2. Massage: In Zone I very light mobilizing massage is given to the affected finger with the arm kept ig elevation. In Zones II - V, circular light to def] friction massage is given. Olive oil is used as tris lubricating medium. If it is too slippery, the oil may be rubbed off for the latter part of the massage. The position of arm elevation for treatment is important (See Fig. 4). Not only will this facilitate the reduction of any swelling but also the relaxation of the patient so necessary for the execution of passive movements. Remember also that the superficial circulation of the hand is easily occluded. It is thus important that any pressure exerted by the physiotherapist's fingers during massage, passive movements and PNF techniques should be relieved frequently during the execution of these techniques.

3. PNF: for all treatments, the patient is best placed supine. The elbow is flexed against resistance to $90^{\circ}$. The physiotherapist then uses her free hand to apply the specific techniques mentioned above, all of which produce very satisfactory results. Rhythmic stabilizations can be done with one hand by using the fingers and palms as alternate resisting surfaces. At a later stage in treatment, full arm PNF techniques are used. Straight arm techniques achieve better results with FDP sutures, whilst pattems 


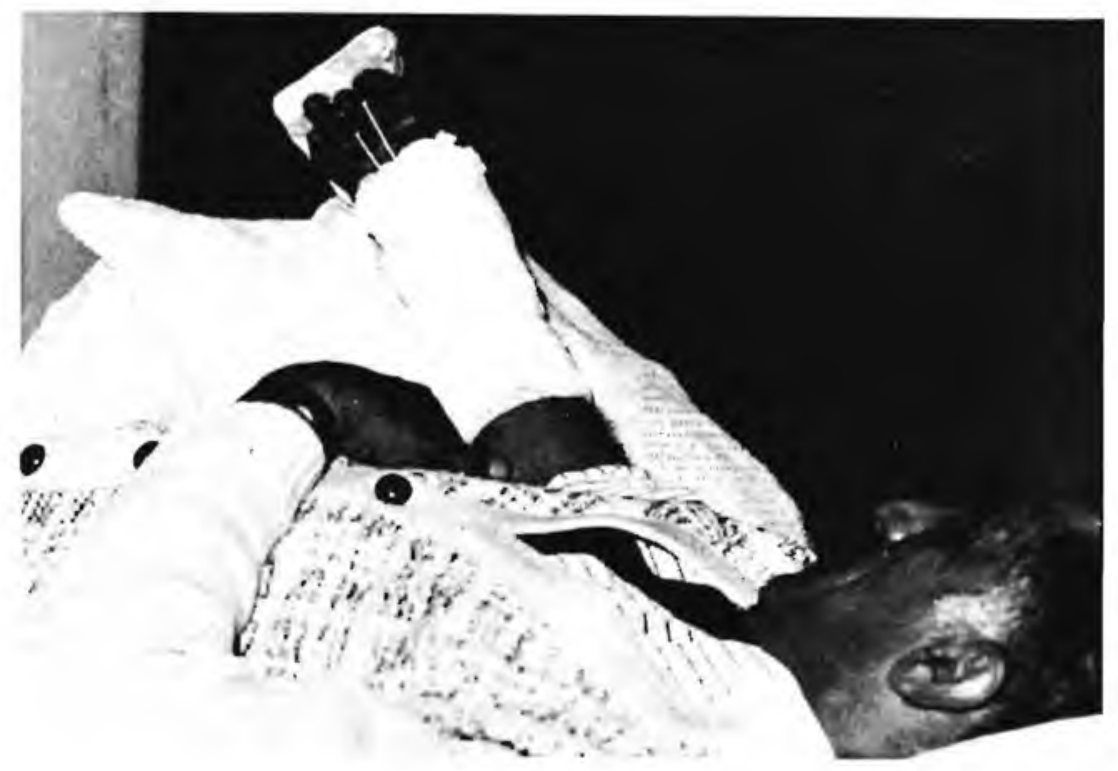

Fig. 4. Treatment in elevation.

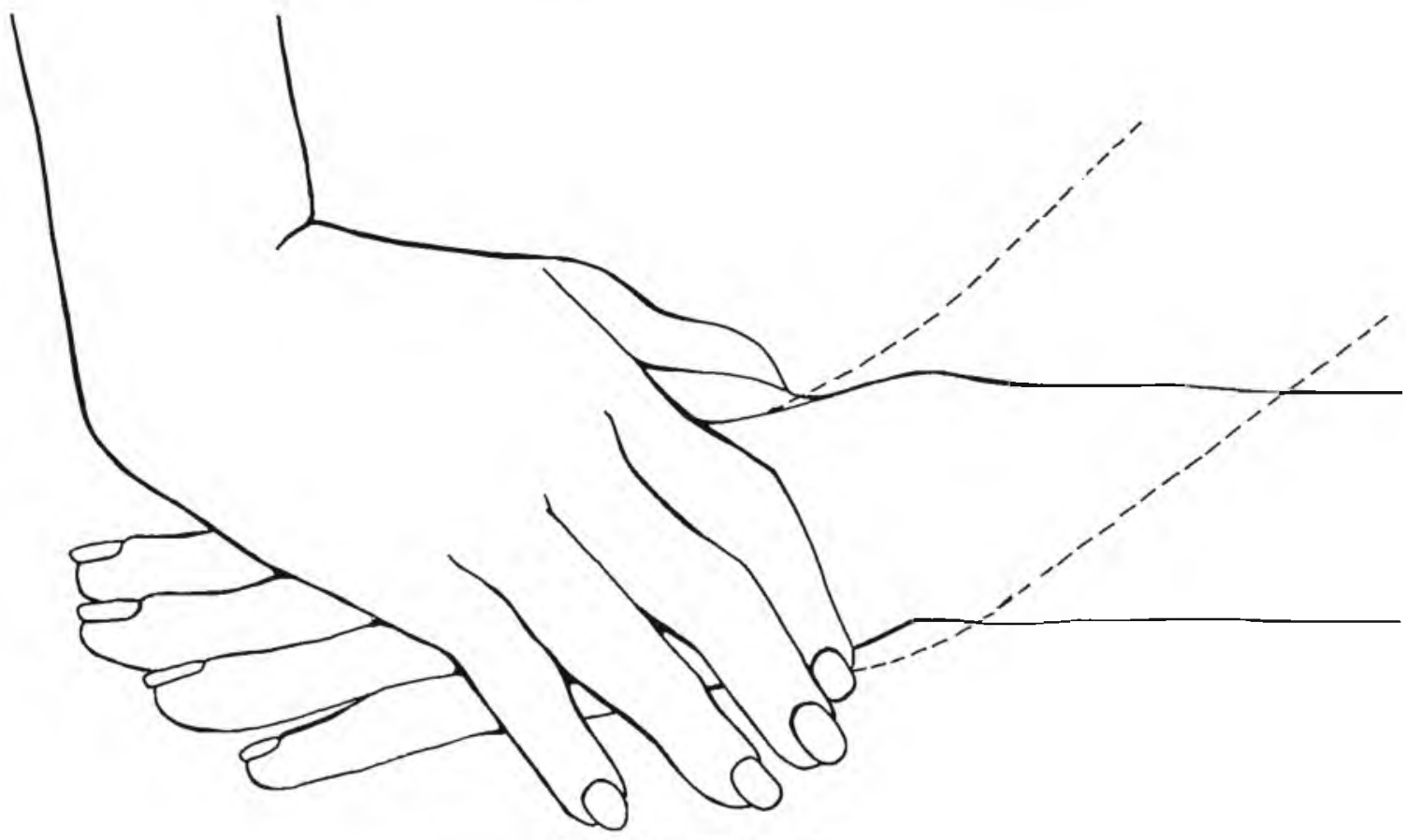

Fig. 5. Passive stretching.

with elbow flexion facilitate better FDS function.

4. Ultrasound is given with increasnig intensities, 0.5 $2 \mathrm{~W} / \mathrm{cm}^{2}$ continuous or $1,5-3 \mathrm{~W} / \mathrm{cm}^{2}$ pulsed. For sutures in Zone $I$ and $I I$ it is best given under water where possible. Ultrasound and massage often produce the best mobilization of adhesions.

5. Passive stretching: This must be carefully executed at first and the force increased gradually. Patients can do their own passive stretching at home from the eighth week onwards by placing the flat hand on a smooth. hard surface (table) and using the other hand to prevent the IP or MP joints from bowstringing', gradually bringing up the wrist to a more extended position by bringing the body weight more vertically over the hand (Fig. 5).

With some FDP sutures in Zone 1 , a certain extensor lag is permissible, since a short portion of the tendon is sacrificed during the operation. This defi- 

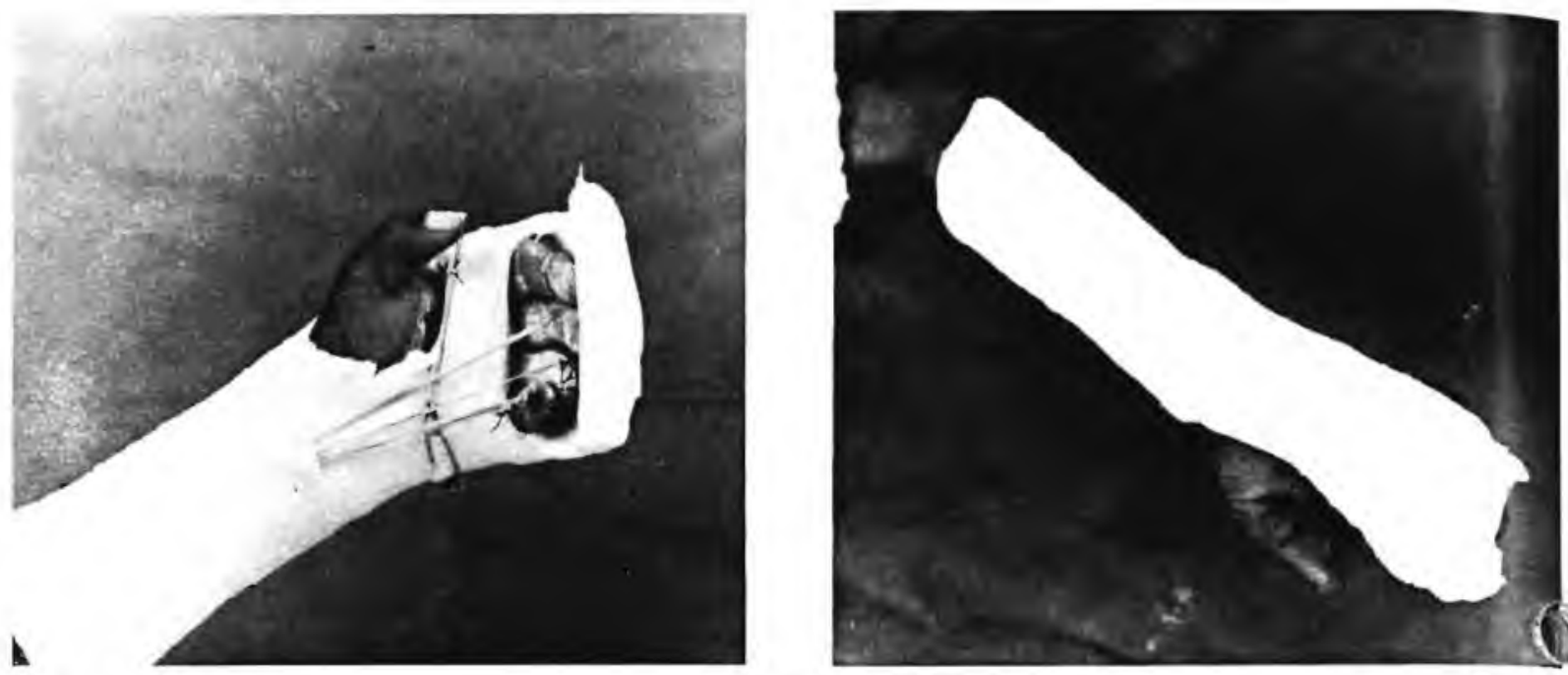

Fig. 6. Serial Plaster.

ciency is automatically made good with time

6. Serial plasters: The purpose of these is not to gain range of movement. but to retain the range gained by the physiotherapist during treatment. The plasters should be comfortable and not hurt the patient. Wynn Parry (1973) suggests wearing these plasters between treatments. but because this may discourage daily activities they are used as night splints only. The splints are made from plaster of Paris and are padded. They stretch from below the elbow across the volar aspect of the wrist to beyond the tips of the terminal phalanges. Grooves should be made to accommodate the fingers separately and reinforcement with the plastic sticks obtained from the middle of the plaster bandages is sometimes necessary (See Fig. 6). The splint is kept in position by means of a crêpe bandage or velcro strips.

7. It has been found that very light adhesions around the wrist seldom benefit from very vigorous physiotherapy i.e. frequent passive stretching, serial plasters and faradism under tension. The tendons adherc tightly to each other and to the surrounding skin and scar tissue. Prolonged stretching and hold-rclax techniques thus do little to loosen adhesions. but can lead to necrosis of the tendons which become threadlike at the site of the adhesion through excessive stretching. In fact, the aforementioned physiotherapy techniques mobilize the scar tissue at the cost of overstretching the tendons. Tenolysis after the above physiotherapy techniques has shown that the tendons are so stretched out that they are ineffective for inner range movements. We also know that after application of these drastic measures, tendons may rupture more easily. With very tight adhesions. the best method of treatment is daily (more if necessary) application of ulirasound. massage over the scar tissue. minimal passive stretch and as much active inner range movements as possible. If progress is unsatisfactory. the patient is referred back to the surgeon for a tenolysis.

8. Pain may limit the achicvement of full range of movement. Passive joint mobilization techniques, Grade III according to Maitland. may then be used to relicve the pain. These techniques are also applied to relieve any 'treatment pain' that may occur.

9. Kaltenborn passive joint mobilizing techniques are not usually necessary. but since patients are often unreliable and stay away. a certain amount of joint stiffness is inevitable and the joint stiffness responds to this form of treatment. Stiff MP joints. however, remain a nightmare! Even the most vigorous techniques are often unsuccessful. The bost method of treatment is, of course, prevention of this stiffness.

\section{Bibliography}

I. Byrne, J. J. (1959): The Hand: Is Allalomy and Diseases. Oxford, Blackwell Scientific Publications.

2. Boyes. J. H. (1964): Bumell's Surery of lhe Hand. 4th Ed. Philadelphia. J. B. Lippincoli.

3. Kaltenborn, F. H. (1974): Manmal Therapy for the Erremity Joinis. Oslo. Olaf Norlis Bokhandel.

4. Maitland. G. D. (1977): Peripheral Manipalarion. 2nd Ed. London. Butterworths. 